\title{
Gauge Invariance of Degenerate Riemannian Metrics
}

\section{Alice Barbara Tumpach}

\section{Introduction}

Having applications to Form recognition in mind, we want to be able to compare shapes of surfaces in $\mathbb{R}^{3}$ in a way that does not depend on parameterizations. To accomplish such so-called gauge invariance, we defined a metric on the space of parameterized surfaces that is degenerate in the direction of reparameterization. ${ }^{1}$

What are the surfaces under consideration? The surfaces we will consider in this note are surfaces which are diffeomorphic to the unit sphere. In other words, the unit sphere will be our model surface, and the surfaces we will consider will be those that can be modeled out of it. To be mathematically precise, these are orientable genus-0 smooth compact surfaces or, equivalently, orientable 2dimensional compact simply connected submanifolds of $\mathbb{R}^{3}$ and will be called spherical surfaces in this note.

How is the unit sphere represented? The good thing about the unit sphere is that only one chart suffices to cover it almost completely. We will use spherical coordinates, with polar angle $\theta$ being greater than 0 (North Pole) and less than $\pi$ (South Pole) and azimuthal angle $\phi$ being greater than or equal to 0 (Greenwich prime meridian) and less than $2 \pi$ (Greenwich prime meridian again); see Figure 1.
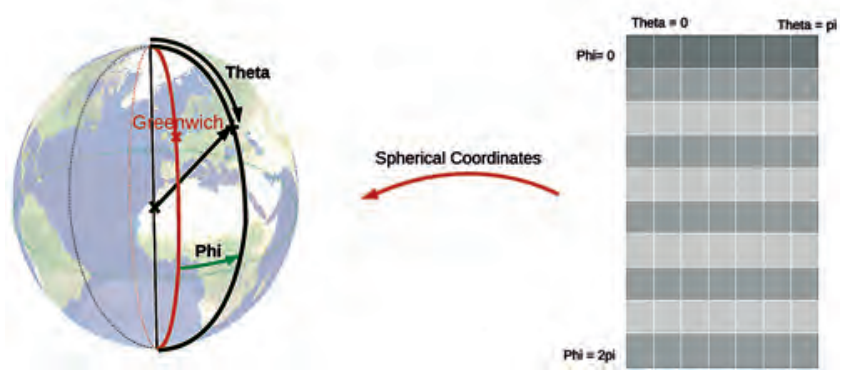

Figure 1. Spherical coordinates.

A. B. Tumpach is associate professor of mathematics at the University Lille 1, France, and member of the Laboratoire Painlevé (Lille 1/CNRS UMR 8524) since 2007. Her email address is Barbara.Tumpach@math. univ-1i11e1.fr.

${ }^{1}$ This note is based on joint work [1] of the author with H. Drira, M. Daoudi, and A. Srivastava.

For permission to reprint this article, please contact: reprintpermission@ams . org.

DOI: http://dx.doi.org/10.1090/noti1350

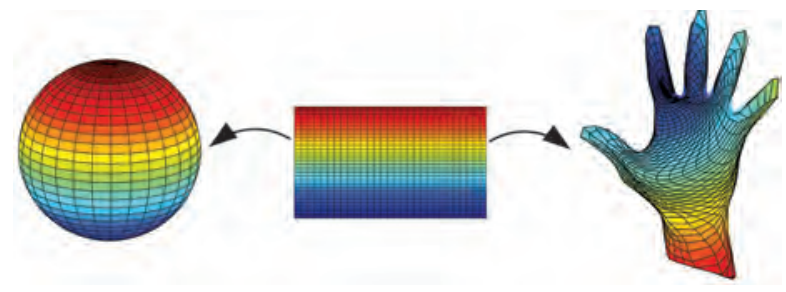

Figure 2. Parameterization of the sphere and of a hand.

What is a parameterization? What we mean mathematically by a parameterization of a spherical surface is a diffeomorphism from the unit sphere to this surface. In practice, however, we will need a discrete version of this notion: it will be a well-behaved mapping from $n \times p$ grid points on the sphere to $n \times p$ points in $\mathbb{R}^{3}$. The distinction is that we will never have a formula for the diffeomorphism, but just the values taken by the diffeomorphism on the vertices of a spherical grid. In order to be able to speak to a computer, we will label the vertices of our favorite spherical grid (for instance, the one given by uniformly placed points on a $2 \pi$-by- $\pi$ rectangle using spherical coordinates; see Figure 2) using two indices ranging from 1 to $n$ for the latitudes and from 1 to $p$ for the longitudes. A computer version of a parameterization is now a 3 -sheeted $(n, p)$-matrix, one sheet for each coordinate $x, y$ and $z$ of $\mathbb{R}^{3}$, containing the coordinates $x_{i j}, y_{i j}$ and $z_{i j}$ of the $\mathbb{R}^{3}$-point associated to the spherical point with indices $(i, j)$.
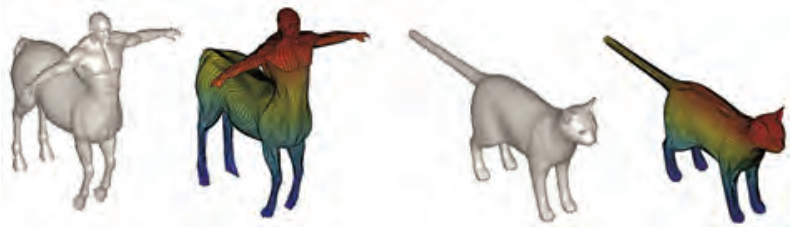

Figure 3. Some triangulated surfaces from the Tosca dataset and their parameterized versions.

How is a surface parameterized? Usually the surfaces we would like to compare do not come with a formula. These are objects of real life, and, unlike the sphere, it may be difficult to make them fit some equations. A 3Dscanner may help give a triangulation of the surfaces we are interested in (i.e. a set of vertices and edges), but it is still a lot of work to build a parameterization out of it. The surfaces used in this paper come from the dataset Tosca tosca.cs.technion.ac.i1/book/resources_data. 

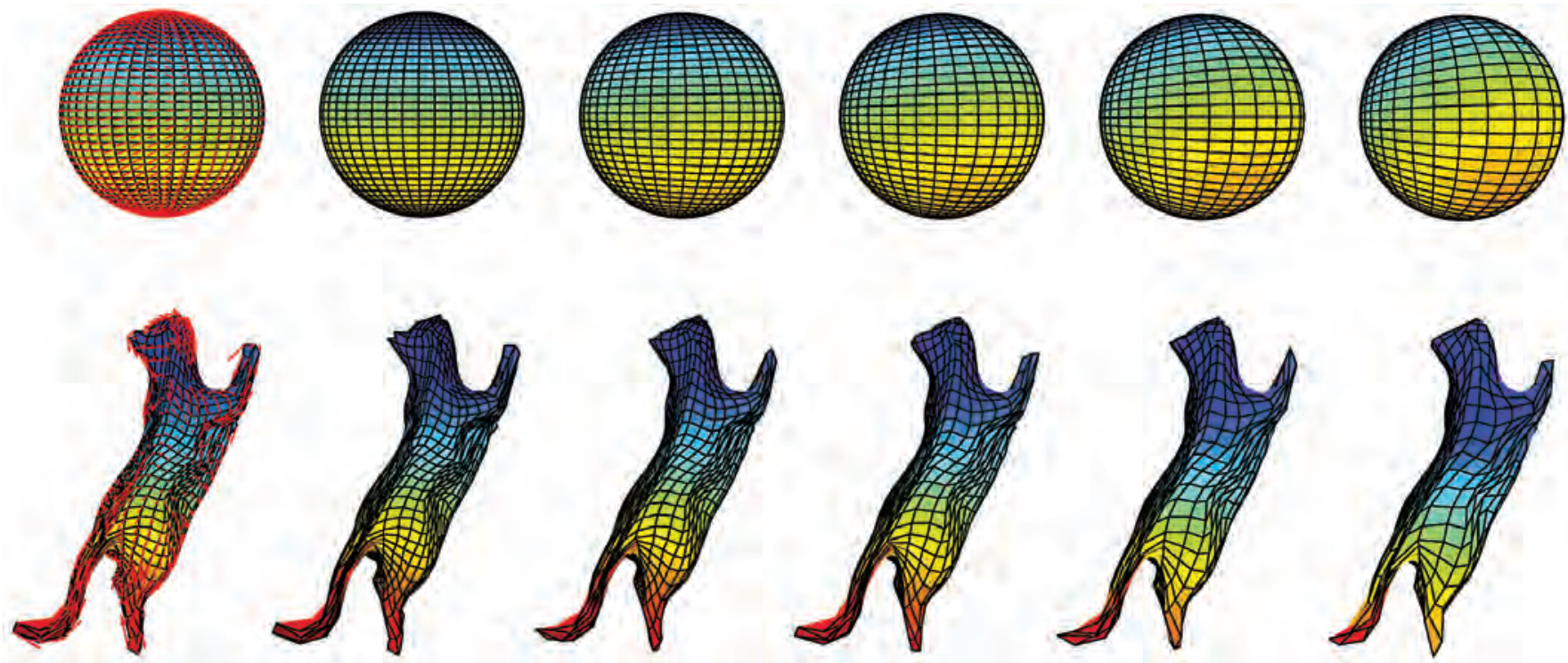

Figure 4. A vector field on the sphere (upper left), and a path of diffeomorphisms having this vector field as velocity at $t=0$ ( 5 other spheres). Bottom line: action of this path of diffeomorphisms on a cat and corresponding vector field.

htm1), containing triangulated surfaces parameterized by H. Laga in [2] (see Figure 3).

How can one visualize a diffeomorphism? One can think of a diffeomorphism of the sphere as a bijection from the sphere to itself mapping smooth curves to smooth curves, tangent spaces to tangent spaces. To visualize a diffeomorphism, one can draw a grid on the sphere and look at how the diffeomorphism moves the grid points. The infinitesimal version of a smooth diffeomorphism is a smooth vector field: at each surface point there is a velocity vector attached which says in which direction and with which amplitude the point has to move. In fact, the set of all smooth (orientation-preserving) diffeomorphisms of the unit sphere, denoted by $\operatorname{Diff}^{+}\left(\mathbb{S}^{2}\right)$, forms a (Fréchet) manifold whose tangent space at the identity map is the space of smooth vector fields. Moreover, this manifold structure is compatible with the group operation given by the composition law, making $\operatorname{Diff}^{+}\left(\mathbb{S}^{2}\right)$ into a Fréchet Lie group. In the upper line of Figure 4, we have depicted a path of diffeomorphisms starting at the identity map and the corresponding velocity vector field at $t=0$. To generate this picture, we used the following family of Möbius transformations :

$$
\phi(t)=\exp t\left(\begin{array}{cc}
-0.05 & 0.5 \\
0.5 & 0.05
\end{array}\right)=\left(\begin{array}{ll}
a(t) & b(t) \\
c(t) & d(t)
\end{array}\right),
$$

where the unit sphere is identified with $\mathbb{C} \cup\{\infty\}$ under the stereographic projection (see Figure 5 ) and where $\phi(t)$ acts on $\mathbb{C} \cup\{\infty\}$ by

$$
z \mapsto \frac{a(t) z+b(t)}{c(t) z+d(t)} .
$$

Note that these diffeomorphisms do not preserve the North and South Poles, hence do not preserve the chart given by the spherical coordinates.

How does a diffeomorphism act on a spherical surface? Given a parameterization of a spherical surface, any

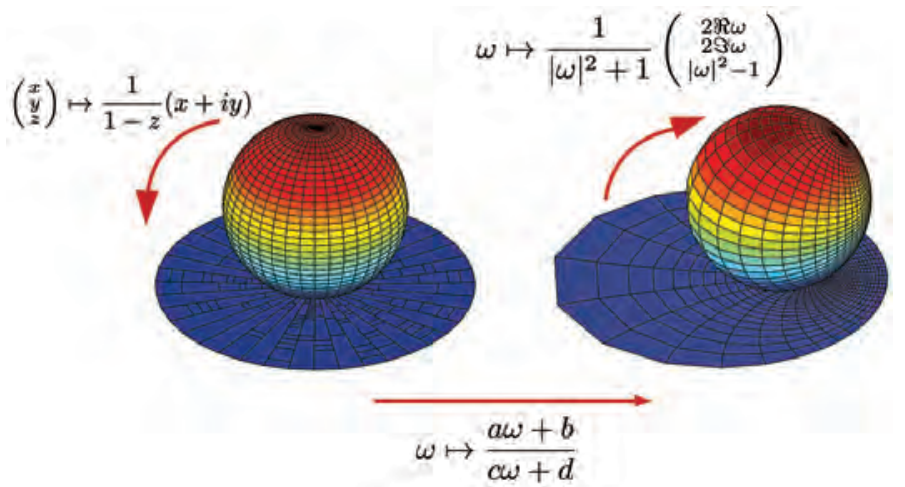

Figure 5. Stereographic projection and Möbius transformation.

diffeomorphism of this surface can be obtained by precomposing the parameterization by a diffeomorphism of the sphere. In this sense, the group of diffeomorphisms $\operatorname{Diff}^{+}\left(\mathbb{S}^{2}\right)$ acts on a parameterized surface by changing its parameterization. Recall, however, that we do not have an explicit formula for the parameterization of most of the surfaces we are interested in, but only the values of the parameterization at some grid points. We therefore need to use some interpolation function (we used the MATLAB $^{\mathrm{TM}}$ function interp2) in order to approximate the values of the parameterization on the spherical grid obtained after applying a diffeomorphism on the sphere. In the bottom line of Figure 4, we have depicted the path of diffeomorphisms of a jumping cat obtained by precomposing our initial parameterization by the path of diffeomorphisms of the sphere illustrated in the upper line of Figure 4.

Is there a preferred parameterization of a spherical surface? Yes, there is. Note that the surfaces we are considering are sitting in the Euclidean ambient 3- 

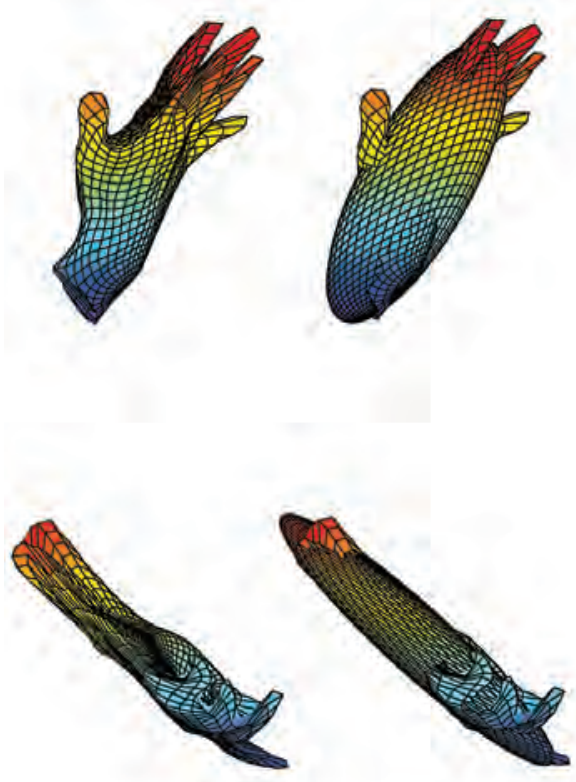
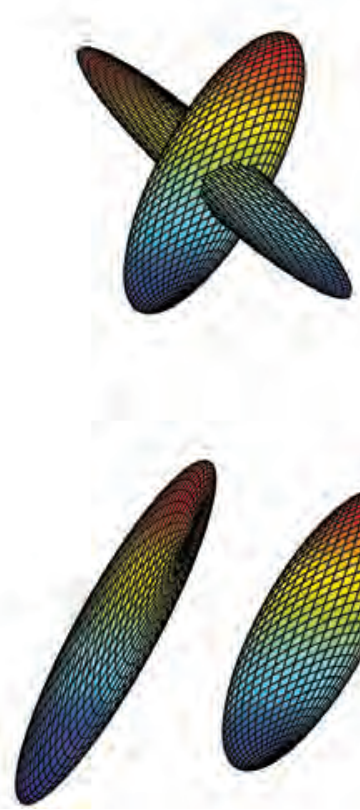

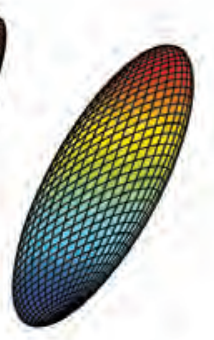

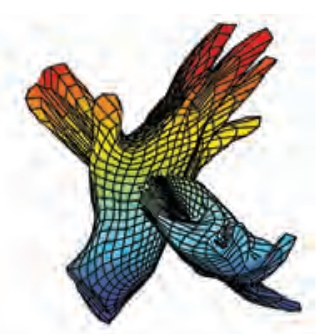

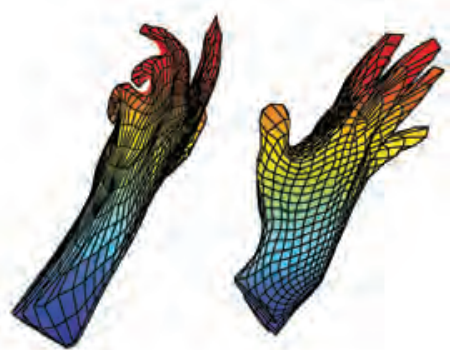

Figure 6. Rotational alignment: two hands before and after the alignment (right column). Each hand is approximated by an ellipsoid (first 2 columns from the left). The rotation used takes the axes of one ellipsoid to the axes of the other (middle column).

dimensional space. It follows that each tangent space at a given point of a spherical surface (for example, at the tip of the middle finger depicted in Figure 7) can be identified with a 2-dimensional vector subspace of $\mathbb{R}^{3}$ to which the Euclidean scalar product of $\mathbb{R}^{3}$ can be restricted. The smoothness of the surface then ensures that these 2-dimensional scalar products on the tangent spaces vary smoothly along the surface, defining what is called a Riemannian metric on the surface. It follows that on a spherical surface one is able to measure angles between two tangent vectors anchored at the same surface point: this angle is exactly the angle between these tangent vectors seen as vectors in $\mathbb{R}^{3}$ (see Figure 7 ). One can also measure distance, in the same way we are measuring distances on Earth, by measuring the shortest path drawn on Earth's surface (and not inside!) joining two given points. In this context, saying that a spherical surface is orientable means exactly that one can define on the surface a unit normal vector field pointing outside the surface. This is enough to ensure that the surface is naturally endowed with a complex structure, the complex structure in a given tangent space being nothing but the rotation of Euclidean angle $+\pi / 2$ around the normal (the orientability helps define the direction of rotation in a coherent way; see Figure 7). In other words, the surfaces we are considering are Riemann surfaces. Since they are compact and simply connected, the uniformization theorem says that they are conformally equivalent to the unit sphere. This means that, given a spherical surface, there exists a homeomorphism, called the uniformization map, which preserves the angles and transforms the unit sphere into the surface. In particular, the uniformization map transforms the coordinate grid into a grid that also has the property of orthogonal intersections (for the orthogonality of vectors in $\mathbb{R}^{3}$ ). Note that the parameterization of the hand given in Figure 2 is not conformal, since it does not preserve the orthogonality of the grid. In fact, given a spherical surface, there are many conformal maps from the unit sphere to it, as many as elements in PSL $(2, \mathbb{C})$. This may sound like a lot, since there are infinitely many complex 2-by-2-matrices with determinant 1 (and the $P$ in $P S L(2, \mathbb{C})$ divides only this amount by $2)$, but $\operatorname{PSL}(2, \mathbb{C})$ is just a 3-dimensional complex Lie

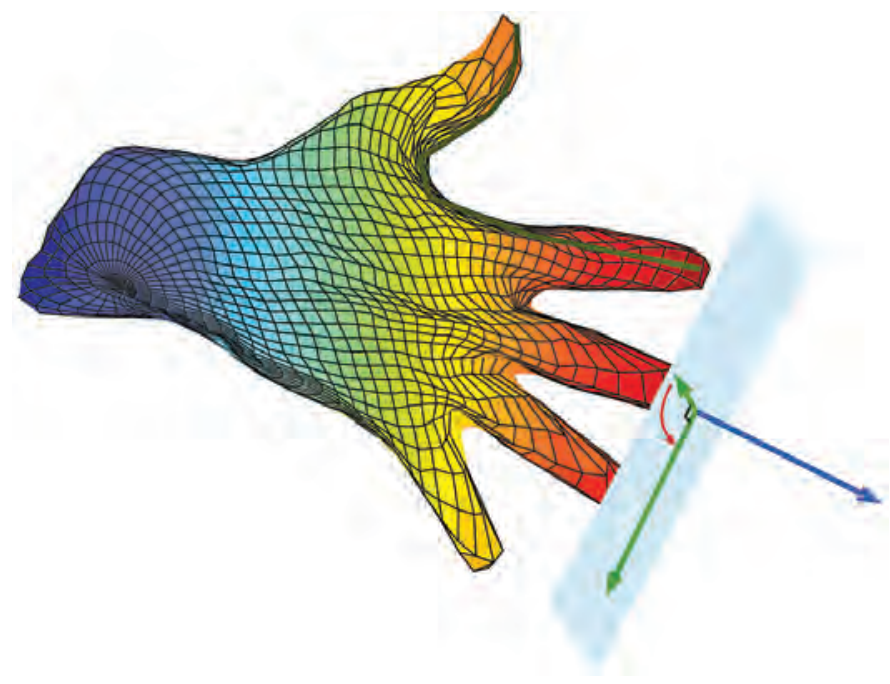

Figure 7. Scalar product on the tangent plan to the tip of the middle finger of a hand, and shortest path from the tip of the index finger to the tip of the thumb. 
group, as opposed to the infinite-dimensional Fréchet Lie group $\operatorname{Diff}^{+}\left(\mathbb{S}^{2}\right)$. Hence, to the question if there exists a preferred parameterization of a spherical surface, a geometer will answer: Yes, modulo PSL(2, $\mathbb{C})$, there is a unique one.

So why not use this preferred parameterization? Because it is hard to implement...

\section{Shape Analysis}

If we want to compare shapes in $\mathbb{R}^{3}$, the first thing to do is to state clearly what is relevant in the shape and what is not. Depending on our situation, one may, for instance, think of a shape as a surface modulo rotation and/or modulo translation and/or modulo scaling. Before comparing two surfaces, one may therefore want to align them properly first and do so in a way that does not depend on the parameterizations. In the next section we explain how the first and second moments of the surface can help us do that. In the section "Fiber Bundle Structure of Preshape Space” we explain the fiber bundle structure of the space of parameterized spherical surfaces. In the section "Characterization of a Shape", we will explain what characterizes a shape. In the section "Gauge Invariance and Riemannian Metrics", we will use this characterization to define a Riemannian metric on the space of shapes and, using the section "Fiber Bundle Structure of Preshape Space", implement it in a way that is independent of the parameterizations (the reader interested in implementing this section can consult [1], where precise algorithms are given).

\section{Alignment of Two Surfaces}

In most situations it makes sense to think of our spherical surfaces as boundaries of 3D-volumes (the surface of a cat has a meaning for us, precisely because it encloses a cat). In order to scale a given surface, we will therefore compute the enclosed volume $V$ and divide each coordinate of surface points by $V^{1 / 3}$. Accordingly, to center a surface, we will compute the center of mass of the enclosed volume and substract it from the coordinates of surface points. The center of mass, whose coordinates are the first moments of the surface, is defined by the following integral over the enclosed volume:

$$
C=\int\left(\begin{array}{l}
x \\
y \\
z
\end{array}\right) \mathrm{dVol} .
$$

In order to rotationally align our spherical surface, we will compute the best ellipsoid that approximates the enclosed volume and apply to the surface points the rotation that maps the axes of the ellipsoid (with decreasing lengths) to the reference axes. This rotation is uniquely defined if the approximating ellipsoid is triaxial (i.e. the lengths of its principal axes are distinct). As an example, Figure 6 shows two hands that have different orientations in space, the corresponding ellipsoids, and the hands after rotation (with a gap to separate them in order to facilitate visualization).

What is the best ellipsoid that approximates a surface? What we expect from the approximation of a surface by an ellipsoid is at least that if we start with an ellipsoid, then it returns the ellipsoid itself. We expect also that if we change the parameterization of the surface, the ellipsoid's shape does not change. To fulfill both conditions, we will need the second moments of the surface defined as the following integral over the enclosed volume:

$$
M=\int\left(\begin{array}{ccc}
x^{2} & x y & x z \\
x y & y^{2} & y z \\
x z & x y & z^{2}
\end{array}\right) \mathrm{dVol} .
$$

The resulting matrix is a symmetric real matrix, hence can be diagonalized in an orthonormal basis. Its eigenvectors define the rotation we are looking for (more precisely, its inverse). To illustrate the robustness of the approximating ellipsoid under reparameterization, we show in Figure 8 different parameterizations of a cat (middle) obtained by precomposing a given parameterization by a diffeomorphism of the sphere (bottom) and the resulting ellipsoid (top).

How are the first and second moments of a surface computed? Recall that, given a spherical surface, we do not have any formula for a parameterization of it. Moreover, we have only a finite number of points on the surface. The integration procedure is therefore replaced by the sum over the oriented tetrahedra defined by two edges on the surface and a surface point (see Figure 11). Recall that the volume of a tetrahedron built on three vectors, $v_{1}, v_{2}$ and $\nu_{3}$, reads $\frac{1}{6} \operatorname{det}\left(v_{1}, v_{2}, v_{3}\right)$. It is important to keep track of the orientation of the surface (in Figure 11, the volume of the red tetrahedron comes with a + sign, whereas the volume of the blue one comes with a - sign). The value of the integral of a polynomial function on a tetrahedron can be expressed (exactly) using just the values taken by the polynomial at a finite number of points on the tetrahedron. For instance, the integral of $x^{2}$ over the tetrahedron with vertices $0, v_{1}=\left(x_{1}, y_{1}, z_{1}\right), v_{2}=\left(x_{2}, y_{2}, z_{2}\right)$, and $v_{3}=\left(x_{3}, y_{3}, z_{3}\right)$ is the volume of the tetrahedron multiplied by $\frac{1}{20} \times\left[\left(x_{1}+x_{2}\right)^{2}+\left(x_{2}+x_{3}\right)^{2}+\left(x_{1}+x_{3}\right)^{2}\right]$.

\section{Fiber Bundle Structure of Preshape Space}

In the introduction we stressed the distinction between the set of all (aligned) parameterized spherical surfaces, called preshape space, and the set of all (aligned) spherical surfaces, called shape space. Recall that the group $\operatorname{Diff}^{+}\left(\mathbb{S}^{2}\right)$ of (orientation-preserving) diffeomorphisms of the unit sphere acts on the preshape space simply by reparameterization. It is noteworthy that two parameterized surfaces correspond to the same surface if and only if one can precompose the first parameterization by a diffeomorphism of the sphere to obtain the second parameterization. One can therefore put an equivalence relation on the preshape space by saying that two parameterized surfaces are equivalent if and only if they can by related by an element of the group $\operatorname{Diff}^{+}\left(\mathbb{S}^{2}\right)$, i.e. if and only if they represent the same surface. The equivalence classes are also called the orbits of the group $\operatorname{Diff}^{+}\left(\mathbb{S}^{2}\right)$ acting on preshape space. Note that two distinct orbits do not intersect; therefore the set of orbits fibers the preshape space in a nice way. There is a one-to-one correspondence between the set of orbits and the shape space. One says that the shape space is the quotient space of the preshape space by the action of the group of diffeomorphisms 

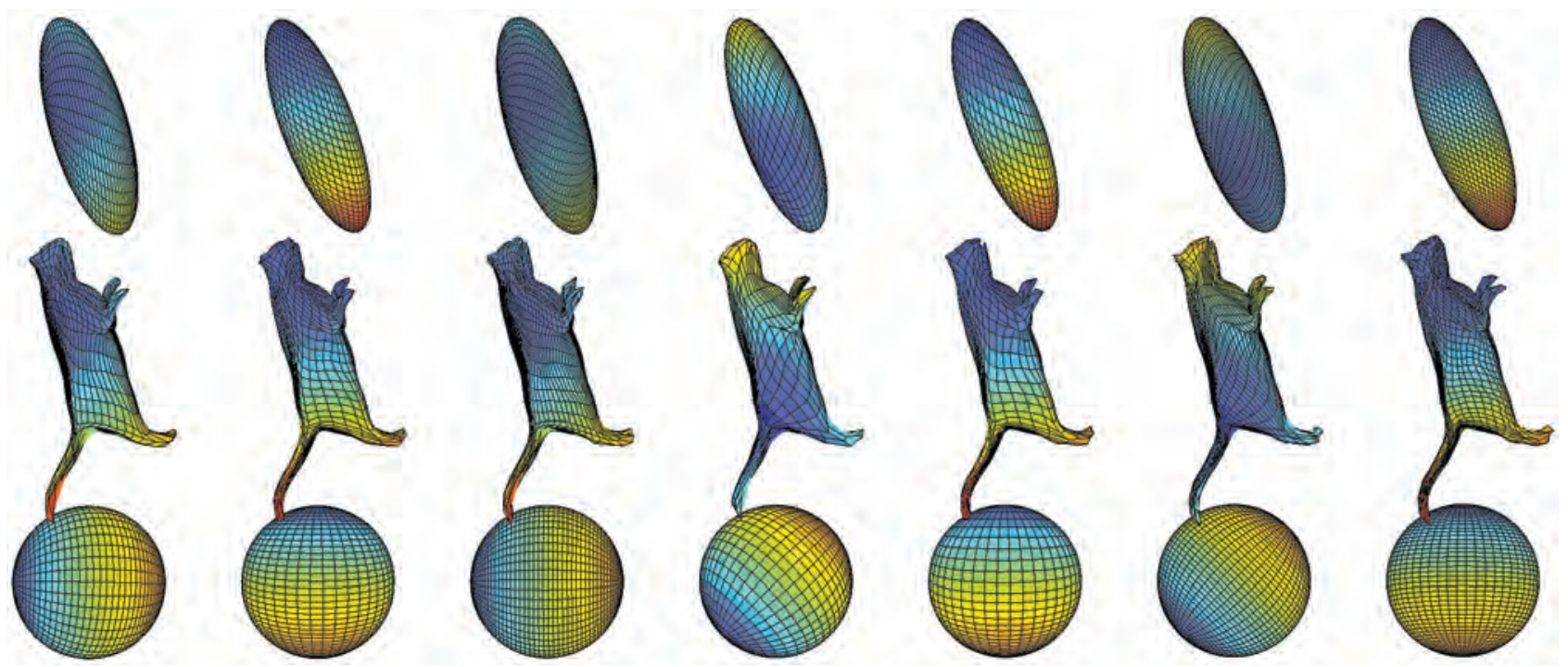

Figure 8. Robustness of the approximating ellipsoid of a surface with respect to reparameterizations.

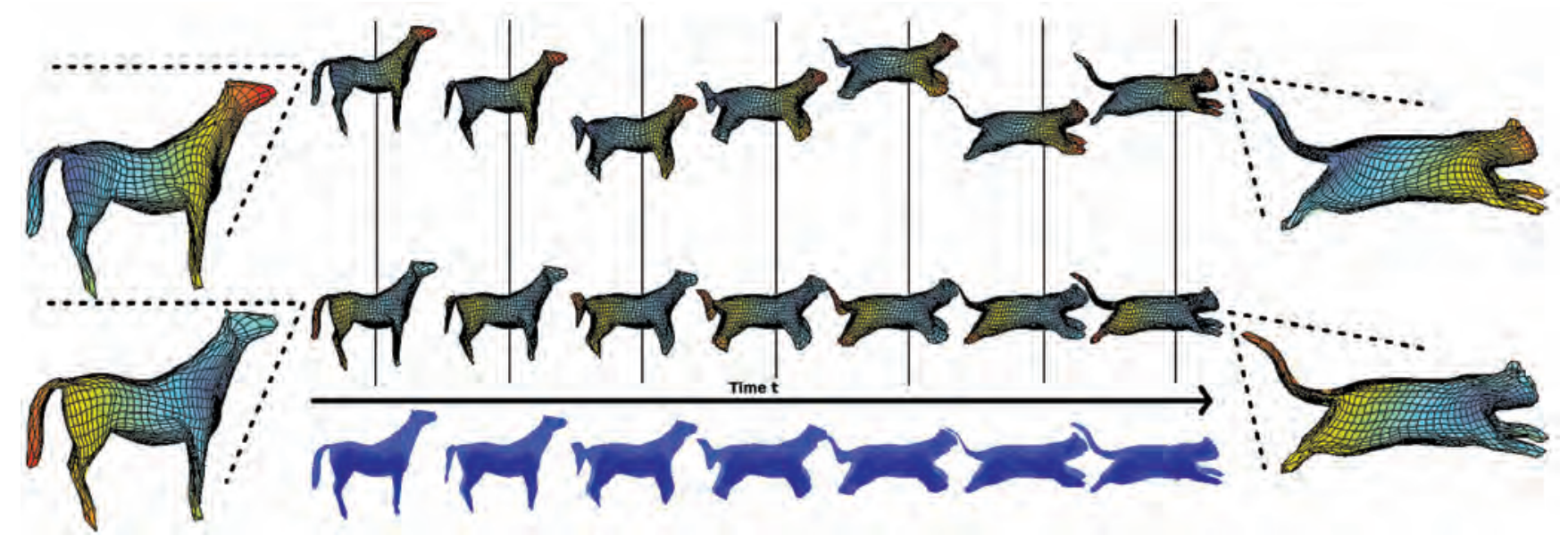

Figure 9. Two paths in preshape space with the same sequence of shapes but with different parameterizations of the corresponding shapes.
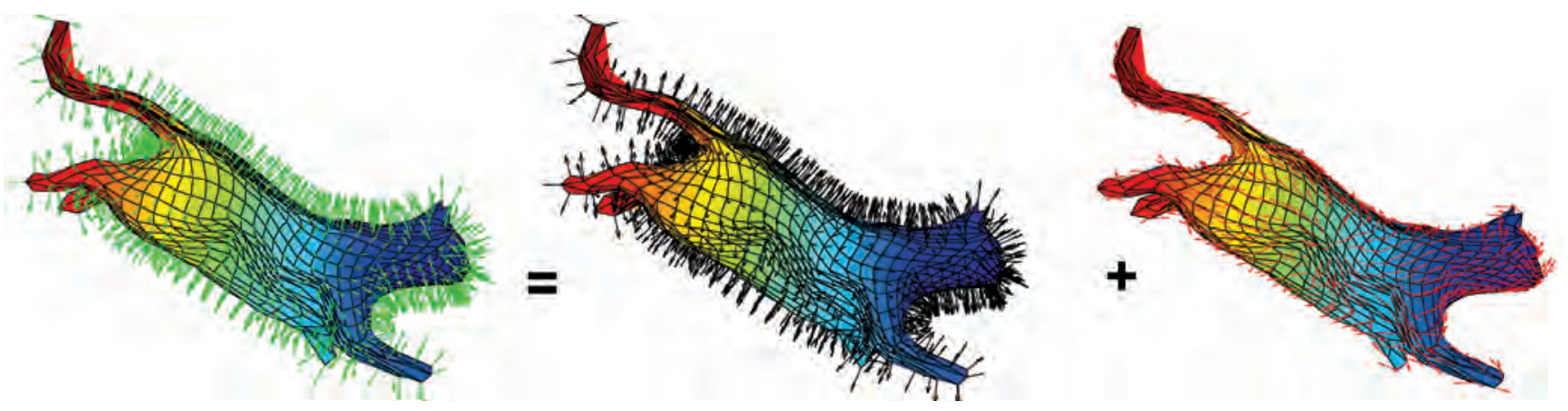

Figure 10. Decomposition of a vector field on the cat (green) into a vector field orthogonal to the cat (black) and a vector field tangent to the cat (red).

of the sphere. In Figure 9 we have illustrated this fiber bundle structure: the blue surfaces in the bottom line are elements in the shape space (no parameterization), and the vertical lines above them symbolize the correspond- 


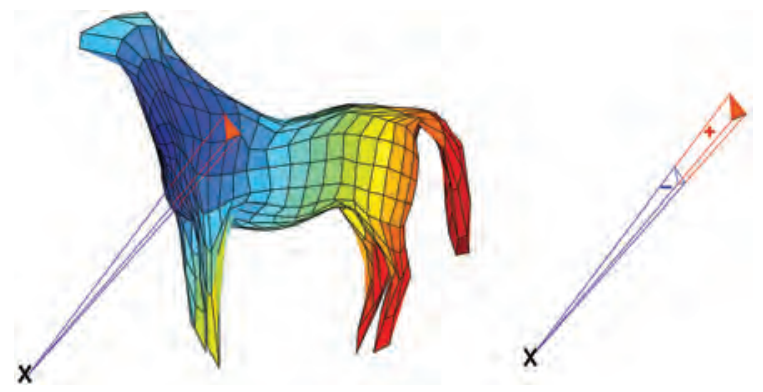

Figure 11. Integration over a triangulated surface.

ing fibers in preshape space. Two elements in each fiber are depicted; for instance, in the first left fiber one can see two parameterized horses that correspond to the same shape.

The preshape space is a smooth (Fréchet) manifold, meaning that locally it looks like a vector space, in the same sense that the earth looks locally like a plane. In fact, the preshape space is an open set in the vector space $C^{\infty}\left(\mathbb{S}^{2}, \mathbb{R}^{3}\right)$ of smooth maps from the unit sphere into $\mathbb{R}^{3}$. Moreover, the fiber bundle structure described above is a smooth one, meaning in particular that the tangent space at some preshape point (which can be identified with $C^{\infty}\left(\mathbb{S}^{2}, \mathbb{R}^{3}\right)$ itself) can be decomposed into the tangent space to the fiber passing through this point and some complement. Since we are dealing with surfaces embedded in $\mathbb{R}^{3}$, there is a natural complement to the tangent space of the fibers (in mathematical terminology, there is a natural connection on this fiber bundle). Indeed, let us describe the tangent space of the fiber at some preshape point, for instance at the parameterization of the cat depicted in Figure 4. By definition, a tangent vector to the fiber passing through this parameterized cat is the velocity vector at $t=0$ of a smooth curve drawn in the fiber whose initial point at $t=0$ is precisely the parameterized cat we are considering. Such a smooth curve is depicted in the bottom line of Figure 4 and is obtained by the action on the parameterized cat of a smooth curve in the diffeomorphism group of $\mathbb{S}^{2}$ starting at the identity (upper line of Figure 4). Hence the tangent space to the fiber passing through the parameterized cat is the space of tangent vector fields to the surface of the cat. A natural complement to this tangent space in $C^{\infty}\left(\mathbb{S}^{2}, \mathbb{R}^{3}\right)$ (which can be identified with the space of $\mathbb{R}^{3}$ valued vector fields on the cat using the parameterization at hand) is the space of vector fields which are orthogonal to the surface of the cat for the scalar product of the Euclidean space $\mathbb{R}^{3}$. In Figure 10 we have depicted the decomposition of an element in $C^{\infty}\left(\mathbb{S}^{2}, \mathbb{R}^{3}\right)$ into the sum of a vector field tangent to the cat and a vector field orthogonal to the cat.

\section{Characterization of a Shape}

If we want to compare shapes, as opposed to parameterized surfaces, one has to understand what is characteristic of the shape, i.e. what is independent of the parameterization. Recall that on a spherical surface one can measure distances and angles just because the surface is sitting in the Euclidean 3-dimensional space. This is encoded by the Riemannian metric on the spherical surface obtained by restricting the Euclidean metric of $\mathbb{R}^{3}$ and is called the first fundamental form of the surface. The second fundamental form is encoding how the surface is embedded into $\mathbb{R}^{3}$. The shape operator is defined using the first and second fundamental forms and tells us how the surface is bent in $\mathbb{R}^{3}$. The shape operator is related to the differential of the normal vector field seen as an application, called the Gauss map, from the surface into the unit sphere, assigning to each point of the surface the unit normal vector to the surface at this point (identified with an element of the unit sphere). The eigenvalues of the shape operator at a given point, called principal curvatures, are the minimal and maximal curvatures that a curve, obtained as the intersection of a plane containing the normal at this point with the surface, can have. For instance, the principal curvatures at any point of a plane are both 0 , whereas the principal curvatures at any point of a sphere of radius $R$ are both $1 / R$. It is a remarkable fact observed by Gauss that the product of the principal curvatures (called Gauss curvature nowadays) depends only on the first fundamental form (Theorema Egregium). The half sum of the principal curvatures is the mean curvature and is what is relevant in the formation of soap films.

How is the curvature at some surface point computed? To compute the principal curvatures $\kappa_{1}$ and $\kappa_{2}$ at a given point of a surface, e.g. at the tip of the index finger of the hand depicted in Figure 12, we first compute the normal at this point by averaging the normals of the facets having this point as vertex. A tangent plane is then defined as the plane orthogonal to the normal passing through the point under consideration. A neighborhood of the point is isolated from the surface (we use a 3neighborhood; see second drawing in Figure 12). We then apply a rigid transformation to center the point at the origin and to align the tangent plane with the $x y$-plane (see third drawing,and a closeup in the fourth drawing). After that, we compute the second-order polynomial $P(x, y)=a_{1} x^{2}+a_{2} y^{2}+a_{3} x y+a_{4} x+a_{5} y+a_{6}$, which minimizes the sum $\sum_{i}\left(z_{i}-P\left(x_{i}, y_{i}\right)\right)^{2}$ over the points of the centered and rotated neighborhood. Then, the Gauss curvature at that point is approximated by $K=4 a_{1} a_{2}-a_{3}^{2}$, the mean curvature by $H=a_{1}+a_{2}$, and the principal curvatures by $\kappa_{1}=a_{1}+a_{2}+\sqrt{\left(\left(a_{1}-a_{2}\right)^{2}+a_{3}^{2}\right)}$ and $\kappa_{2}=$ $a_{1}+a_{2}-\sqrt{\left(\left(a_{1}-a_{2}\right)^{2}+a_{3}^{2}\right)}$.

What characterizes a spherical surface? It follows from the fundamental theorem of surface theory that two parameterized (smooth) surfaces $f_{1}$ and $f_{2}$ having the same first and second fundamental forms differ at most by a translation and a rotation. Therefore, in order to characterize an aligned surface, one can use its first and second fundamental forms or, better, its first fundamental form $g$ and its Gauss map $n$. 


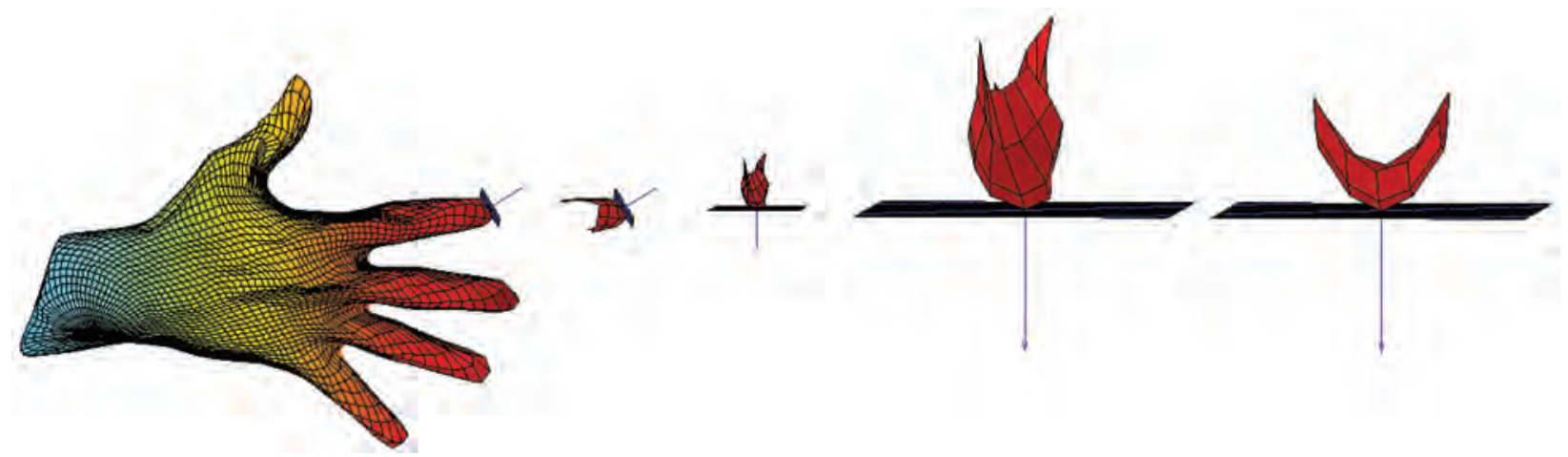

Figure 12. From left to right: A hand with the tangent plane and normal at the tip of the index finger, 3-neighborhood of the tip of the index finger, tip of the index finger after rotation, a closeup, approximating second-order polynomial.

\section{Gauge Invariance and Riemannian Metrics Elastic Riemannian Metric}

Recall that a Riemannian metric on a manifold is a collection of scalar products on the tangent spaces to the manifold, which vary smoothly when one travels along the manifold. In the Introduction, we have seen that the spherical surfaces are naturally Riemannian manifolds when endowed with the restriction of the Euclidean metric of $\mathbb{R}^{3}$. Here we are talking about the same mathematical notion of Riemannian manifold but on a higher level of abstraction: indeed our manifold is now the set of all parameterized spherical surfaces, called preshape space, and the tangent space at a given parameterized surface is the vector space $C^{\infty}\left(\mathbb{S}^{2}, \mathbb{R}^{3}\right)$ (see the section "Fiber Bundle Structure of Preshape Space"). The elastic metric is a (3parameter family of) Riemannian metric(s) on preshape space which quantifies infinitesimal variations of the first fundamental form $g$ and of the normal vector field $n$ according to the following formula in which $\lambda, a, c$ are positive parameters:

$$
\begin{array}{r}
\left\langle\left\langle\delta f_{1}, \delta f_{2}\right\rangle\right\rangle_{f}=\int_{\mathbb{S}^{2}} d s|g|^{\frac{1}{2}}\left\{a \operatorname{Tr}\left(g^{-1} \delta g_{1} g^{-1} \delta g_{2}\right)\right. \\
\left.+\frac{\lambda}{2} \operatorname{Tr}\left(g^{-1} \delta g_{1}\right) \operatorname{Tr}\left(g^{-1} \delta g_{2}\right)+c \delta n_{1} \cdot \delta n_{2}\right\},
\end{array}
$$

where $f: \mathbb{S}^{2} \rightarrow \mathbb{R}^{3}$ is a parameterization of a spherical surface, $\delta f_{i}$ denotes variations of $f, \delta g_{i}$ denotes the corresponding variations of the first fundamental form $g$, and $\delta n_{i}$ denotes the corresponding variations of the normal vector field $n$ (for more information about the use of this metric in computer science see [1]).

\section{Quotient Riemannian Metric}

One way to define a Riemannian structure on shape space is to put a Riemannian metric on preshape space that is invariant by the action of the diffeomorphism group. It is not hard to see that the elastic metric defined in the previous section does have this property. The resulting Riemannian metric on shape space is called the quotient metric.

How is the quotient metric defined? Consider two infinitesimal deformations $X$ and $Y$ of the hand with a missing finger depicted in Figure 13. Choose a parameterization $f$ of this hand (in the fiber $\mathcal{O}_{f}$ above the shape of the hand) and two infinitesimal deformations $\tilde{X}$ and $\tilde{Y}$ of this parameterized hand that project onto $X$ and $Y$. There are many possible choices for $\tilde{X}$ and $\tilde{Y}$, since adding an

infinitesimal deformaWe would like to be tion tangent to the fiber $\mathcal{O}_{f}$ will not change able to measure the length of a metamorphosis between two surfaces in a manner that does not depend on the parameterizations used along the way. the projection onto the tangent space to shape space. But there is a choice which is better relative to the Riemannian metric on preshape space we started with: it is when $\tilde{X}$ and $\tilde{Y}$ are orthogonal to the space $T_{f} \mathcal{O}_{f}$. In that case, the norms of $\tilde{X}$ and $\tilde{Y}$ are minimal (one says that $\tilde{X}$ and $\tilde{Y}$ are horizontal for the connection defined by ric on preshape space) The scalar product of $X$ and $Y$ is then defined as the scalar product of these minimal $\tilde{X}$ and $\tilde{Y}$. The invariance of the metric ensures that the real number obtained this way does not depend on the choice of the parameterization $f$ of the hand.

One drawback in this definition is that usually the minimal $\tilde{X}$ and $\tilde{Y}$ are hard to find, in particular, for the elastic metric defined in the previous section. But there is a second, even more problematic, drawback. Indeed, for the quotient Riemannian metric, two paths of parameterized surfaces projecting to the same path of unparameterized surfaces will have (in general) different lengths. As an example, we have depicted in Figure 9 two deformations from a parameterized horse to a parameterized jumping cat that project to the same sequence of (blue) shapes. Because the elastic metric also measures deformations 


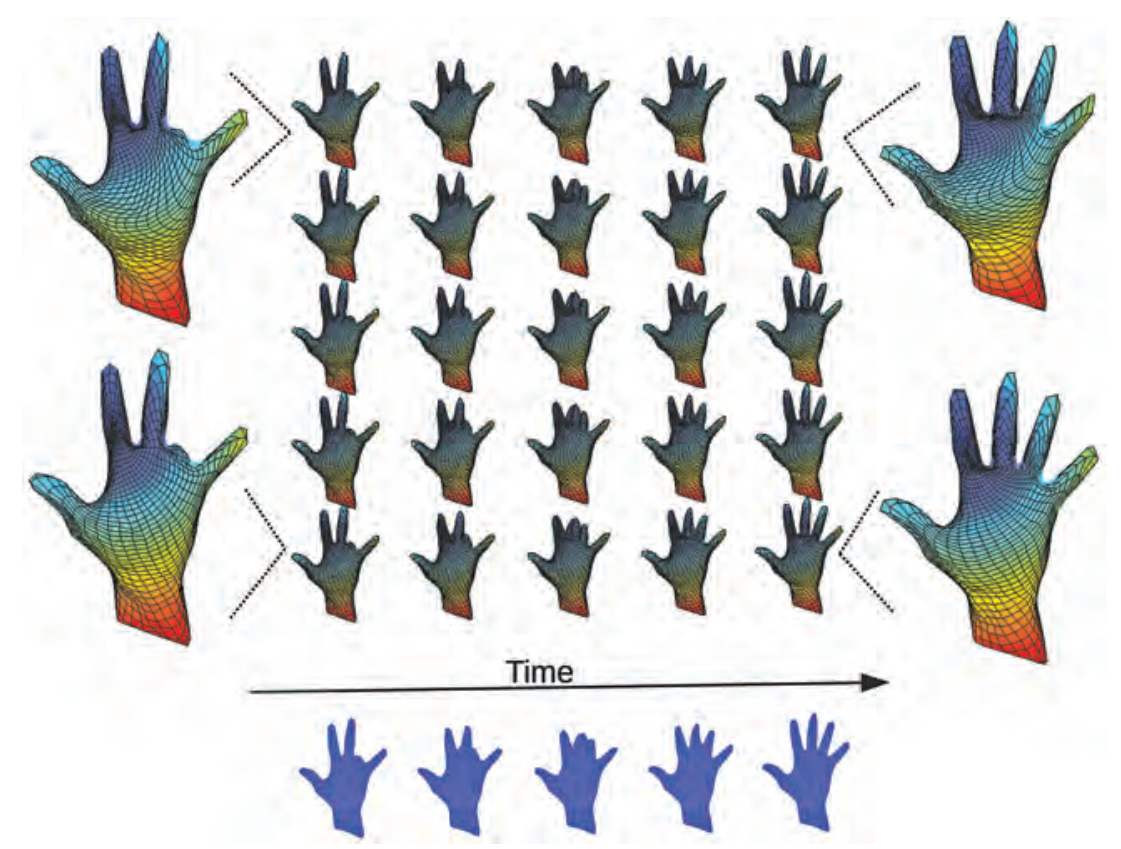

Figure 13. Deformation of hand with a missing finger into a full hand and diverse parameterizations of this deformation.

along the fibers, i.e. variations of the "height" in Figure 9, these two deformations of parameterized surfaces do not have the same lengths in preshape space.

\section{Defining Gauge Invariance}

Contrary to the situation described in the previous section, we would like to be able to measure the length of a metamorphosis between two surfaces in a manner that does not depend on the parameterizations used along the way. This requirement is in the same spirit as the requirement that the length of a road be independent of the speed profile of cars traveling on it (and measuring distances using their internal machinery). More precisely, we would like the length $L[\Psi]$ for any path $\Psi$ in preshape space to match the length of the path $t \mapsto \Psi(t) \circ \gamma(t)$, where $t \mapsto \gamma(t) \in \operatorname{Diff}^{+}\left(\mathbb{S}^{2}\right)$ is any timedependent reparameterization of $\mathbb{S}^{2}$. Formally, the group $G:=C^{\infty}\left([0,1]\right.$, Diff $\left.^{+}\left(\mathbb{S}^{2}\right)\right)$ of time-dependent reparameterizations acts on the space of preshape paths by reparameterizing each shape of the path. The group $G$ is called the gauge group, and one says that $G$ acts by gauge transformations. We are looking for a framework where the length of a path is invariant to gauge transformations.

How is a gauge invariant framework built? The idea is very simple. Considering the preshape space as a fiber bundle over the shape space (see the section "Fiber Bundle Structure of Preshape Space"), recall that the tangent space to the fiber passing through a given parameterized surface $f$, denoted by $T_{f} \mathcal{O}$, has a natural complement defined as the space of normal vector fields to the surface and is denoted by $\mathrm{Nor}_{f}$. Instead of starting with a Riemannian metric on preshape space (for which vector fields tangent to the surfaces have positive norm), we will define a degenerate Riemannian metric on preshape space in the following way. First, we will declare that $T_{f} \mathcal{O}_{f}$ and $\mathrm{Nor}_{f}$ are orthogonal subspaces. Second, we will declare that the scalar product of any two elements in $T_{f} \mathcal{O}_{f}$ is 0 . And last, we will put a $\operatorname{Diff}^{+}\left(\mathbb{S}^{2}\right)$-invariant scalar product on the space of normal vector fields.

Since the tangent space to an unparameterized spherical surface can be identified with the space of normal vector fields (in fact, the space of normal deformations is what is used to define the manifold structure of shape space), this procedure defines a Riemannian metric on shape space (the Diff ${ }^{+}\left(\mathbb{S}^{2}\right)$-invariance ensures that the scalar product of two normal vector fields does not depend on the parameterization of the surface). Moreover, because we have chosen the degeneracy of the metric to match exactly the tangent space to the fibers, the resulting Riemannian metric on shape space is nondegenerate. In fact any Riemannian metric on shape space can be obtained in this way (just reverse the construction).

\section{Gauge Invariant Metric}

It follows from the previous section that in order to define a gauge invariant (degenerate) Riemannian metric on preshape space, it is sufficient to specify the scalar product of two normal vector fields, the only requirement being that this scalar product be invariant by the action of the diffeomorphism group. Since the elastic metric defined in the section "Elastic Riemannian Metric "is Diff ${ }^{+}\left(\mathbb{S}^{2}\right)$ invariant, one can, for instance, use its restriction to the space of normal vector fields. The resulting (nondegenerate) Riemannian metric on shape space can be expressed using the principal curvatures $\kappa_{1}$ and $\kappa_{2}$ and the first 
fundamental form $g$ in the following way:

$$
\begin{aligned}
((h n, k n))_{S}=\int_{S} d S\{h k & \left(2 a\left(\kappa_{1}-\kappa_{2}\right)^{2}\right. \\
& \left.\left.+2(\lambda+a)\left(\kappa_{1}+\kappa_{2}\right)^{2}\right)+c \delta n_{1} \cdot \delta n_{2}\right\},
\end{aligned}
$$

where $S$ is any spherical surface, $h$ and $k$ any real functions on $S$, and $n$ the unit normal vector field on $S$ pointing outward. The difference $\kappa_{1}-\kappa_{2}$ in the first term has been called the normal deformation of the surface. The sum $\kappa_{1}+\kappa_{2}$ is twice the mean curvature that measures variations of the area of local patches. These two terms are related to the shape index idx $=\frac{2}{\pi} \arctan \frac{\kappa_{1}+\kappa_{2}}{\kappa_{1}-\kappa_{2}}$. The last term in equation (2) measures variations of the normal vector field, i.e. bending.

Note that this Riemannian metric on shape space differs from the quotient metric defined in the section "Quotient Riemannian Metric". In fact, quotient metric and gauge invariant metric coincide if and only if Nor $_{f}$ is orthogonal to $T_{f} \mathcal{O}_{f}$ for the Riemannian metric on the preshape space we started with, a property that is not satisfied by the elastic metric (1).

\section{Conclusion}

What is the advantage of a gauge invariant metric? Two paths in preshape space that project to the same path in shape space may have different lengths in the Riemannian setting but the same length in the present framework. For instance, in Figure 13 any two paths that project onto the metamorphosis of a growing finger depicted in the bottom line have the same length in the gauge invariant setting. The two paths depicted in Figure 9 from a parameterized horse to a parameterized jumping cat have the same length (as computed by our programs; see [1]). Moreover, a path in preshape space which consists just in reparameterizing a shape, as for example the vertical paths in Figure 13, will have nonzero length in the Riemannian setting but zero length for the degenerate gauge-invariant Riemannian metric on preshape space. For more information on how to use this framework for automatic classification of shapes, we refer the reader to the original paper [1].

\section{Acknowledgments}

This note was written during a visit of the author at the Pauli Institute, Vienna, Austria, where the programs used in this note were implemented using a MATLAB license of the University of Vienna. This work was also supported in part by the Labex CEMPI (ANR-11-LABX0007-01). The author would like to thank F. Gueritaud for reading this manuscript and for his valuable comments and suggestions.

\section{References}

[1] A. B. Tumpach, H. Drira, M. DaOudi, and A. Srivastava, Gauge invariant framework for shape analysis of surfaces, 2015, to appear in IEEE Transactions on Pattern Analysis and Machine Intelligence, Institute of Electrical and Electronics Engineers.
[2] S. Kurtek, A. Srivastava, E. Klassen, and H. Laga, Landmark-guided elastic shape analysis of sphericallyparameterized surfaces, Eurographics 32 (2013), no. 2.

\section{About the Author}

Alice Barbara Tumpach received a $\mathrm{PhD}$ degree in mathematics in 2005 at the École Polytechnique, Palaiseau, France. She spent two years at the École Polytechnique Fédérale de Lausanne as a postdoc. Her research interests lie in the areas of infinitedimensional geometry, Lie groups, and functional analysis. She gives master's courses on Lie groups and organizes conferences on infinite-dimensional geometry for the Federation of Mathematical Research of Nord-PasCalais, France. She also acts in videos for Exo7, available on YouTube, where she explains basic notions of linear algebra. She is currently on sabbatical in order to raise her three kids and devotes with pleasure the free time she has left to mathematical research. 